\title{
Importância dos mecanismos de investimento para implantação de novos negócios: um estudo ao redor do arco metropolitano
}

\section{The importance of investment mechanisms for setting up new businesses: a study around the metropolitan arc}

DOI: $10.46814 / 1 a j d v 3 n 4-031$

Recebimento dos originais: 01/05/2021

Aceitação para publicação: 31/06/2021

\author{
Rodrigo Rodrigues de Freitas \\ Doutorando em Engenharia de Transporte \\ Centro Federal de Educação Tecnológica Celso Suckow da Fonseca CEFET/RJ, Departamento \\ de Engenharia de Produção \\ Rod. Gov. Mário Covas, s/n - Santana, Itaguaí - RJ, 23812-101 \\ E-mail: rodrigo.freitas@ cefet-rj.br

\section{Diego Manoel Enedino Gonçalves} \\ Mestrando em Engenharia Nuclear \\ Universidade Federal do Rio de Janeiro, Programa de Engenharia Nuclear PEN/COPPE/UFRJ \\ Av. Horácio Macedo, 2030, Bloco G - Sala 206 - CT, Cidade Universitária CEP. 21.941-914 - \\ Rio de Janeiro - RJ \\ E-mail: diegomanoelgoncalves@gmail.com

\section{Thiago de Souza Cardoso Mayrink Paiva} \\ Engenheiro Mecânico \\ Centro Federal de Educação Tecnológica Celso Suckow da Fonseca CEFET/RJ, Departamento \\ de Engenharia Mecânica \\ Rod. Gov. Mário Covas, s/n - Santana, Itaguaí - RJ, 23812-101 \\ E-mail: thiagoscmpaiva@gmail.com
}

\section{RESUMO}

O trabalho realizado possui o propósito de aumentar as possibilidades na tomada de decisão, a luz de uma abordagem financeira em relação a implantação de terminais logísticos ao redor da BR 493/RJ arco metropolitano. As oscilações e choques externos demandam uma abordagem probabilística, a fim de, flexibilizar a tomada de decisão e mitigar o risco inerente a projetos expostos a volatilidade de mercado. A aplicação do modelo de Black \& Scholes em opções reais possibilita a absorção de choques externos e mitigação do risco inerente a precificação do ativo analisado. Confrontando o modelo anterior, utilizamos o modelo de Geske ajustado por Kemna para opções composta. Observando que ambas são opções europeias e possuem duas possibilidades de investimento inicial para a tomada de decisão.

Palavras chaves: Terminal logístico, Análise de investimento, Opções reais.

\begin{abstract}
The research aims to increase the possibilities in decision making, in light of a financial approach regarding the implementation of logistics terminals around the BR 493 / RJ - metropolitan arc. External oscillations and shocks require a probabilistic approach in order to make decision making more flexible
\end{abstract}


and mitigate the risk inherent to projects exposed to market volatility. The application of the Black \& Scholes model in real options allows the absorption of external shocks and mitigation of the risk inherent to the pricing of the analyzed asset. Confronting the previous model, we used the Geske model adjusted by Kemna for composite options. Noting that both are European options and have two possibilities of initial investment for decision making.

Key words: Logistics terminal, Investment analysis, Real options.

\section{INTRODUÇÃO}

Em uma economia globalizada, caracterizada por mudanças constantes, empresas enfrentam dilemas diários, a fim de se estabelecerem em mercados cada vez mais inovadores e competitivos. Diante da necessidade de tomar grandes decisões em períodos relativamente curtos, análises de caráter econômico-financeiro são fundamentais para garantir a competitividade e sobrevivência de uma empresa, fornecendo ao gestor uma estimativa dos efeitos das decisões em momentos futuros que podem influenciar diretamente na tomada de decisão hoje.

Segundo Minardi (2004), a flexibilidade gerencial de um projeto financeiro é apenas uma possibilidade, e não uma obrigação, porém os acréscimos de possibilidades melhoram a tomada de decisão e suas relações com investidores. Devido ao grau de incerteza presente nos projetos, o constante aperfeiçoamento nos métodos de análise de investimento é fundamental para garantir uma visão mais refinada e fiel à realidade, bem como a escolha da metodologia a ser utilizada para lidar com a incerteza presente no tipo de investimento desejado. Segundo Lee (2011) a opção está vinculada a incerteza do projeto, pois ao contrário do fluxo de caixa descontado (FCD), onde o valor do investimento se deprecia com o aumento da volatilidade, o método TOR valoriza a flexibilidade gerencial como uma opção incorporada. Investimentos para a implantação ou abertura de uma empresa de logística em novas áreas como a região da BR 493/RJ - Arco Metropolitano demandam altos valores iniciais devido a necessidade de investir em infraestrutura o que reforça a importância do uso de ferramentas de análise de investimentos mais complexas, capazes de captar fatores como incerteza e flexibilidade administrativa (Ribeiro, 2020).

O relatório Rima (2007), o Arco Rodoviário da Região Metropolitana do Rio de Janeiro - Arco Metropolitano, na rodovia federal BR 493, situado entre os municípios de Duque de Caxias na BR 040 (Rio - Juiz de fora) e Itaguaí na BR 101 atende ao tráfego de longa distância oriundo das regiões SUL/SUDESTE em direção às regiões NORTE/NORDESTE do país conectando as rodovias federais atravessadas, BR-040, BR-116 (Norte e Sul) e BR-465 e BR-101 (Norte e Sul), em um posicionamento estratégico do porto de Itaguaí em sua primeira fase. De acordo com o projeto de implantação, a justificativa relacionada com o presente trabalho é viabilizar a implantação de terminais logísticos, com 
redução dos tempos de viagem e custos de transportes, bem como a distribuição destas cargas para os mercados consumidores e introduzir novas possibilidades de expansão urbana para os municípios localizados próximos à rodovia.

A pesquisa propõe a utilização de ferramentas financeiras de análise de projeto para auxiliar na tomada de decisão para abertura de novos negócios em ambientes logísticos. Segundo Copeland e Antikarov (2003), uma opção é o direito, mas não a obrigação, de tomar uma ação (Ex. adiar, expandir, contratar ou abandonar) a um custo predeterminado chamado preço de exercício, durante um determinado tempo. Após a introdução, a segunda sessão tem por objetivo justificar a motivação para a realização desta pesquisa, a terceira sessão apresenta os métodos e modelos utilizados, na quarta sessão deste artigo temos a aplicação e os resultados obtidos através dos métodos e modelos matemáticos previamente introduzidos, e por fim, a quinta sessão contempla a conclusão e os resultados obtidos.

\section{MÉDOTO E AMBIENTE DE INCERTEZA}

A pesquisa tem por finalidade simular a implantação de um terminal logístico situado na região do arco metropolitano apenas por ferramentas de análise econômico-financeiro aplicado a métodos de análise de investimentos sob e incerteza. A princípio, os métodos tradicionais de análise de investimento como é o caso do FCD, não satisfazem uma pesquisa de longo prazo, pois ao longo do tempo, a economia e os indicadores financeiros sofrem perturbações externas e oscilações temporais. Segundo Dixit e Pindyck (1994) o método do valor presente líquido - VPL ou FCD não conseguem absorver o valor da flexibilidade, pois não consegue se antecipar ao risco previsto, ocorre principalmente por que utiliza de desconto uma taxa constante como na Eq. 1, logo esse método é utilizado para análise livre de risco, uma vez que, a taxa de desconto é constante no tempo sem absorção dos riscos inflacionários. O FCD é orientado para ambiente com baixa oscilação da taxa de juros, partindo dessa premissa, optou-se pela aplicação da Teoria das Opções Reais - TOR, devido a sua capacidade de incorporar incertezas ao valor do projeto, além de precificar o valor da opção, auxiliando no processo de tomada de decisão.

Segundo Dixit e Pindyck (1994), o fluxo de caixa descontado (FCD) é uma ferramenta para projetar o valor atual de uma empresa ou investimento, porém não reconhece a flexibilidade administrativa como planejamento estratégico. Na análise do fluxo de caixa em longo prazo, percebese duas categorias específicas: reversível e irreversível. A categoria reversível assume que os gastos podem ser recuperados, caso as condições de mercado mudem ou fiquem piores que o esperado (Santos e Pamplona, 2001). A categoria irreversível assume uma posição de agora ou nunca sem permitir uma flexibilidade na tomada da decisão, assim como o método do FCD. 


$$
V P L=- \text { Investimento } \sum_{t=0}^{\infty} \frac{F C}{(1+i)^{n}}
$$

A assimetria de informações distorce os mercados na relação investimento versus confiabilidade, por isso existem alguns modelos na utilização de tomada de decisão, na qual, cria uma flexibilidade administrativa. Segundo Minardi (2000) e Trigeorgis (1993), a assimetria resultante criada pela adaptabilidade requer uma regra para um VPL expandido que reflita os dois valores componentes: o VPL tradicional (estático ou passivo) e o valor da opção de operação e adaptabilidade estratégica. Dessa forma, tem-se:

$$
\left.V P L_{\text {expandido }}=V P L_{\text {estático ou passivo }}+\text { Valor da opção (flexibilidade administrativa }\right)
$$

Neste trabalho é sugerido precificar a flexibilidade administrativa através da utilização do modelo de Black \& Scholes (1973), lembrando que esse modelo fornece apenas o valor da opção como uma adição ao valor da empresa. Contudo, aplica-se o modelo de precificação de compra para continuar com o empreendimento após o investimento para produção. Assim, como o modelo de precificação de venda, caso o empreendimento seja vendido pelo valor esperado de mercado, após o término do projeto ou antes do investimento para produtividade. No primeiro caso, se a opção de compra for maior que o investimento inicial, a operação está coberta, logo, pode-se optar pela continuação do negócio, ao contrário, vende o empreendimento. O segundo caso, se utilizar a opção de venda, e o valor for menor que o investimento inicial, a operação não está coberta, logo não se pode optar pela venda do empreendimento.

Confrontando com o modelo anterior, será aplicado o modelo de Geske (1979) como opção composta, no caso de ocorrer adiamento do projeto, ou outra necessidade de mais um período de investimento. Esse modelo é ajustado para avaliação de opções reais por Kemna (1993) na Eq. 3, na qual, incorpora o valor a partir do segundo investimento, gerando um valor total de retorno do projeto.

$$
\text { Modelo de GESKE = opções composta - Investimento inicial }
$$

\section{METODOLOGIA APLICADA}

A abordagem busca modificar o senso comum, e aprofundar em uma investigação mais elaborada onde a proposta foi elaborar um procedimento racional e sistemático para buscar respostas aos problemas propostos. A avaliação da TOR acrescenta uma flexibilidade administrativa, segundo Santos e Pamplona (2002), a opção financeira paga-se um direito, e não uma obrigação de exercê-la 
até a data do vencimento (opção americana), se exercida na data do vencimento (opção europeia) deve estar previamente estabelecido em contrato, uma vez que, o risco assumido oscila ao longo do tempo.

Para justificar o período para implantação de um novo negócio ao longo do Arco Metropolitano, a luz da taxa livre de risco - TLR, foram realizadas duas análises de sensibilidade aplicando o método de Black \& Scholes (1973) e Geske (1979), ajustado para avaliação de opções reais por Kemna (1993). Deve-se utilizar o modelo de Geske, somente quando o projeto obtiver uma característica de duas opções de períodos para decisões de investimento antes de produzir, vender ou abandonar. O método de Black \& Scholes compreende a aplicação da opção de compra e opção de venda e o modelo de Geske compreende um suporte para a tomada de decisão em opções compostas.

\subsection{MODELO DE BLACK \& SCHOLES}

- Opção de compra:

$$
C=S_{0} * N\left(d_{1}\right)-E_{c} e^{-r t} * N\left(d_{2}\right)
$$

Em que:

$\mathrm{C}=$ Preço da opção de compra.

$\mathrm{S}=$ Preço do ativo real atual.

$\mathrm{Ec}=$ Valor do investimento em produtividade.

$r=$ Taxa livre de risco.

$\mathrm{t}=$ Tempo para $\mathrm{o}$ vencimento da opção, em anos.

$\sigma=$ Volatilidade do preço do ativo, expressa ao ano.

$\mathrm{N}(.$.$) = Função de distribuição normal acumulada.$

Onde:

$$
d_{1}=\left(\ln \left(\frac{S}{E}\right)+\left(r+\frac{\sigma^{2}}{2}\right) T\right) / \sigma \sqrt{T}
$$

$\mathrm{e}$;

$$
d_{2}=d_{1}-\sigma \sqrt{T}
$$

- Opção de venda

$$
P=E_{v} e^{-r t} N\left(-d_{2}\right)-S_{0} N\left(-d_{1}\right)
$$


Em que:

$\mathrm{P}=$ Preço da Opção de Venda.

$\mathrm{S}=$ Preço do ativo real atual.

$\mathrm{Ev}=$ Preço de venda do projeto $\mathrm{E}$ (valor de mercado).

$\mathrm{r}=$ Taxa livre de risco.

$\mathrm{t}=$ Tempo para o vencimento da opção, em anos.

\subsection{MODELO DE GESKE (APLICAÇÃO DE OPÇÕES COMPOSTA)}

$$
G=F e^{-r t} M(k, h, \rho) M\left(k-\sigma \sqrt{t^{*}}, k-\sigma \sqrt{t} ; \rho\right)-K^{*} e^{-r t} N\left(k-\sigma \sqrt{t^{*}}\right)
$$

Em que:

$\mathrm{F}=$ Valor presente do fluxo de caixa;

$\sigma=$ Volatilidade histórica do dólar;

$\mathrm{K}=$ Investimento para produtividade;

$\mathrm{K}^{*}=$ Investimento de follow-up (aporte depois do investimento inicial);

$\mathrm{r}=$ taxa livre de risco;

$\mathrm{t}=$ segundo período (compreende o período do investimento em produtividade);

$\mathrm{t}^{*}=$ primeiro período (compreende o período do investimento de follow-up)

$\mathrm{Fc}=$ Valor crítico do projeto (onde a primeira opção será exercida);

$\mathrm{M}=$ Distribuição normal bivariada cumulativa;

$\mathrm{N}$ = Distribuição norma padrão.

Cálculo de $\rho$, h e k:

$$
\begin{gathered}
\rho=\left(\frac{t^{*}}{t}\right)^{1 / 2} \\
h=\frac{\ln (F / K)+\frac{1}{2} \sigma^{2} t}{\sigma \sqrt{t}} \\
k=\frac{\ln \left(F / F_{C}\right)+\frac{1}{2} \sigma^{2} t^{*}}{\sigma \sqrt{t^{*}}}
\end{gathered}
$$

Último termo destacado no modelo de Geske (1979) é o valor crítico (Fc), pois é a primeira opção de valor que o investidor exercer para aceitar ou não o projeto. Utiliza-se a abordagem de Dixit e Pindyck (1994), relacionando o Fc em relação ao valor presente líquido, valor da opção e o investimento inicial.

$$
F C=I+F_{0}
$$


Logo,

Para $\mathrm{F}_{\mathrm{c}}$ maior que $\mathrm{S}_{0}$ - I é melhor o investidor não adiar o investimento, contudo, se $\mathrm{Fc}$ for menor que $\mathrm{S}_{0}-\mathrm{I}$, o investidor deverá executar o empreendimento, logo se forem iguais, a decisão é indiferente, optando por outras variáveis.

\section{SENSIBILIDADE DO INVESTIMENTO EM RELAÇÃO A TAXA LIVRE DE RISCO}

A taxa livre de risco é utilizada em alguns modelos de redução de risco para investimento em portfólio como o modelo CAPM (Capital Asset Pricing Model) desenvolvido por Sharpe (1964); Treynor (1961 e 1962 apud Korajczyk, 1999); Lintner (1965); Black (1972). Percebe-se que desde 1992, com o artigo de Fama e French as críticas em relação a publicação, principalmente da dificuldade de provar a correlação entre o retorno e o beta de mercado. Segundo Fama e French (1992) os testes empíricos de 1962 a 1990 não puderam evidenciar correlação positiva entre o retorno e o beta de mercado. Essa condição era o pressuposto básico para a confirmação do modelo, onde Black (1993) fez duras críticas na estimação e interpretação dos dados para estimar o valor de beta. Estudos destacados por Roll e Ross (1994) percebe que uma mensuração dos dados de forma ex-ante ineficiente poderá traçar uma curva dentro da fronteira eficiente e não sobre a fronteira eficiente de investimento em relação ao retorno e risco. Eles afirmam ainda que a regressão do beta poderá determinar um $\mathrm{R}^{2}$ próximo de zero, logo, com baixa correlação ao longo do tempo. Para determinar a melhor estimação dos dados, Copeland et al. (1995) propuseram que a melhor taxa livre de risco seria o retorno de um portfólio com beta igual a zero, já proposto por Black (1972). Ainda Copeland et al. (1995) argumentam que em países em desenvolvimento o modelo CAPM originalmente proposto não é adequado, reiterando que se deve aperfeiçoar o modelo e não o descartar.

A proposta da pesquisa para a taxa livre de risco seria resultado da taxa básica de juros menos a inflação, justamente para propor uma taxa que ao menos, na utilização do valor presente, a taxa absorvesse a inflação do período, capitalizando a sua diferença. A proposta parece razoável, uma vez que Black (1992) e reafirmada por Copeland et al. (1995), a taxa livre de risco seria o retorno de um portfólio com beta igual a zero. Essa proposta procura capitalizar a uma taxa de retorno de um portfólio, onde o beta de mercado oscila na mesma proporção que um título de menor risco econômico.

Como a investigação desse trabalho trata-se de investimento em bens reais, e não no mercado financeiro, utiliza-se a taxa de remuneração de títulos públicos e não uma taxa de remuneração em mercados de capitais. Segundo Sharpe et al. (1999), conforme as características da taxa ser livre de risco, o retorno deve ser fixo, sem possibilidade de não haver o pagamento no vencimento. Contudo, o aumento da taxa básica de juros tende a provocar uma migração de capital do lado real de bens e 
serviços para o lado monetário, esse efeito tende a reduzir os investimentos tangíveis, direcionando para o mercado financeiro, provocando a redução de empregos e do fluxo de moeda para as famílias.

\section{AVALIAÇÃO DE UM PROJETO EM LOGÍSTICA - APLICANDO OPÇÕES REAIS}

\subsection{APLICANDO BLACK \& SCHOLES}

As escolhas dos métodos ocorreram mediante o melhor modelo para a implantação de terminais logísticos, pois o modelo de Black \& Scholes proporciona a opção da flexibilidade administrativa em relação ao resultado estático (VPL), esse modelo é derivado do movimento geométrico browniano, que deriva da cadeia de Markov, acrescido de valores estocásticos, proporciona uma modelagem estatística ex nunc. A pesquisa ocorreu com levantamento de dados financeiros das empresas da região, através de publicações aos acionistas e agências governamentais, logo utilizamos como modelo a Opção de Compra e Opção de Venda para empresas instaladas na zona industrial do município de Queimados.

Tabela 1: Valores médio para implantação do terminal logístico - 2016

\begin{tabular}{|c|c|}
\hline Valor Presente - $2014\left(\mathrm{~S}_{0}\right)$ & $\mathrm{R} \$ 17.071 .383,83$ \\
\hline Valor Presente - 2015 & $\mathrm{R} \$ 19.461 .377,56$ \\
\hline Valor Presente - $2016(\mathrm{~F})$ & $\mathrm{R} \$ 22.185 .970,42$ \\
\hline Fluxo de caixa - 2016 & $\mathrm{R} \$ \quad 3.106 .035,86$ \\
\hline Valor de mercado - 2014 & $\mathrm{R} \$ \quad 3.370 .830,00$ \\
\hline Valor de mercado - 2015 & $\mathrm{R} \$ \quad 3.842 .746,20$ \\
\hline Valor de mercado - $2016(\mathrm{Ev})(\mathrm{E})$ & $\mathrm{R} \$ \quad 4.380 .730,67$ \\
\hline Investimento inicial - 2014 & $\mathrm{R} \$ \quad 2.797 .930,00$ \\
\hline Investimento inicial - 2015 & $\mathrm{R} \$ \quad 3.189 .640,20$ \\
\hline Investimento inicial - $2016(\mathrm{Ec})(\mathrm{K})$ & $\mathrm{R} \$ \quad 3.636 .189,83$ \\
\hline Investimento do ativo/direitos $\left(\mathrm{K}^{*}\right)$ & $572.900,00$ \\
\hline Valor crítico do projeto $(\mathrm{Fc})$ & $\mathrm{R} \$ 11.789 .598,70$ \\
\hline Taxa Básica de Juros & $14 \%$ \\
\hline Taxa livre de risco (a.a) (i) & $6,13 \%$ \\
\hline Volatilidade em $\$ \$(\sigma)$ & $30,00 \%$ \\
\hline Vencimento (anos) $\left(\mathrm{t}^{*}\right)$ & 1 \\
\hline Vencimento (anos) $(t)$ & 2 \\
\hline
\end{tabular}

Os valores da Tabela 1 foram extraídos, a fim de verificar a média do custo de implantação de uma empresa de logística ao redor do Arco Metropolitano. O valor de investimento inicial em 2014 foi proposto baseado nas instalações de um terminal logístico no distrito industrial de Queimados à $42 \mathrm{~km}$ da ilha da madeira onde ficam as principais instalações do Porto de Itaguaí, passando pelo arco metropolitano. Os valores estão capitalizados de acordo com o período proposto e taxa livre de risco no período. A volatilidade é o desvio-padrão dos dados históricos do movimento diário do fechamento 
da moeda Real/Dólar, e por fim, o vencimento é o prazo ajustado entre o início do projeto até a decisão de investir para produzir ou vender, antes de iniciar as atividades. Testes mostraram que quando maior o prazo, aumenta o risco do projeto e o valor da opção, uma vez que os investidores criam uma expectativa de maior retorno no futuro.

Abaixo, o desenvolvimento da análise e a sua relação entre o VPL estático e a flexibilidade administrativa citado na Eq.1.

Substituindo os dados da Tabela 1 na Eq. 1;

$$
V P L=-572.900,00-572.900,00 /(1,14)^{-1}-3.636 .189,83 /(1,14)^{-2}+22.185 .970,00 /(1,14)^{-2}=R \$ 13.198 .009,97
$$

Lembrando que os fluxos foram descontados a taxa básica de juros de $14 \%$ a.a em dezembro de 2016, que será constante no tempo. Utiliza-se do valor presente em perpetuidade referente ao fluxo líquido de caixa de 2016, o resultado do VPL foi de R\$13.198.009,97. Esse cenário representa a irreversibilidade se iniciado em 2014. Como a pesquisa se utiliza de bens reais, opta-se por utilizar a taxa básica de juros, pois estão incorporados à inflação do período e a taxa livre de risco, conforme mencionado. Se o VPL for positivo, aceita o projeto, caso contrário, o projeto não terá aporte de capital. A taxa livre de risco será a taxa básica de juros menos a inflação do período, reiterando que esse modelo é utilizado para economias em desenvolvimento, pois como a taxa básica de juros é utilizada no portfólio para remunerar títulos públicos, ela sempre estará acima da inflação, justamente para proteger o investimento de quem compra, ao contrário, em economias desenvolvidas, Black (1972) e Copeland et al. (1995) orientam utilizar para o mercado dos EUA ou similares (i) o retorno das letras do tesouro americano (treasure bills), (ii) a taxa dos títulos americanos (bonds) de dez anos, e (iii) a taxa dos títulos americanos de trinta anos, dependendo do tempo de aplicação do projeto.

Se os acionistas exigirem um valor menor à R\$ 11.789.598,70 como valor crítico em 2016, o projeto será negado. A tomada de decisão depende do cenário econômico do período, como o FCD é um processo irreversível, dificilmente o projeto poderia ser aproveitado. Contudo o método de opções reais aumenta a possibilidade da tomada de decisão baseada em movimentos estocásticos.

\section{- Opção de Compra}

Segundo Santos e Pamplona (2005), a opção de compra é o valor da flexibilidade administrativa pelo fato do investidor ter o direito, mas não a obrigação de exercê-la, posteriormente, poderão optar pela continuação do projeto. No caso desta pesquisa, o prazo entre o investimento em ativos e o investimento em produtividade (investimento inicial $E c$ ) é observado como opção de diferimento, onde somente poderá ser exercida na data do vencimento, logo será considerada uma opção europeia. 
Substituindo os dados da Tabela 1 nas Eqs. 5 e 6;

$d 1=\left[\ln (17.071 .383,83 / 3.636 .189,83)+\left(0,0613+0,3^{2} * 0,5\right) * 2\right] / 0,3^{*} 2^{1 / 2}=4,146161196$

$$
\begin{gathered}
N(d 1)=0,499983095 \\
d 2=4,146161196-0,3 * 2^{1 / 2}=3,721897127 \\
N(d 2)=0,499901134
\end{gathered}
$$

Substituindo os dados da Tabela 1 e as Eqs. 15 e 17 na Eq.4;

$$
C=17.071 .383,83 * 0,499983095-3.636 .189,83 e^{-0,0613 * 2 * 0,499901134=R \$ 6.927 .402,87}
$$

Utilizando opção de compra em Black \& Scholes obtêm-se o valor da flexibilidade administrativa, comparando com o prêmio (investimento em ativos/direitos). $\mathrm{C}=\mathrm{R} \$ 6.927 .402,87$ > $\mathrm{Iad}=\mathrm{R}$ \$ 572.900,00. Pode-se concluir que a operação está coberta, logo investir em produtividade é uma opção. Onde o valor de $\mathrm{C}$ deve ser adicionado ao projeto.

\section{- Opção de Venda}

Segundo Santos e Pamplona (2005) a opção de venda é o valor acrescido ao projeto pelo fato o investidor ter o direito, mas não a obrigação, de posteriormente, optar pela venda dos direitos. Deve ser vista como uma opção de abandono. Neste caso, o preço do exercício será visto como preço da venda dos direitos (valor de mercado - Ev)

Substituindo os dados da Tabela 1 nas Eqs. 5 e 6;

$$
\begin{gathered}
d 1=\left[\ln (17.071 .383,83 / 4.380 .730,67)+\left(0,0613+0,3^{2} * 0,5\right) * 2\right] / 0,3 * 2^{1 / 2}=3,707097036 \\
N(d 1)=0,0001048824 \\
d 2=3,70709736-0,3^{*} 2^{1 / 2}=3,282832967 \\
N(d 2)=0,000513848
\end{gathered}
$$

Substituindo os dados da Tabela 1 e Eqs. 20 e 22 na Eq. 7; 
$P=4.380 .730,67 * e^{-0,0613 * 2 *(0,000513848)}-17.071 .383,83 *(0,000104824)=R \$ 201,81$

A mesma comparação da opção de compra deve ser feita em opção de venda em Black \& Scholes, logo $\mathrm{P}=\mathrm{R}$ \$ 201,80 < Iad = R \$ 572.900,00. Pode-se concluir que a operação está descoberta, logo investir para depois exercer o direito de vender (opção de abandono) não é uma opção. Pois a flexibilidade administrativa não paga o investimento em ativos e direitos.

De acordo com Minardi (2000) e Trigeorgis (1993) deve-se calcular o VPL expandido para ter o valor do projeto com a flexibilidade administrativa. Utilizando os dados das Eqs. 13, 18 e 23, para opção de compra e venda sucessivamente;

$$
\text { VPL }_{\text {TOR }} \text { - compra }=13.198 .009,97+6.927 .402,87=\quad \mathrm{R} \$ 20.125 .412,84
$$

$\mathrm{e}$

$$
\text { VPL } \text { TOR - venda }=13.198 .009,97+201,80=\mathrm{R} \$ 13.198 .211,77
$$

Percebe-se que utilizando Black \& Scholes, o investidor obtém uma flexibilidade maior na tomada de decisão em relação a aplicação do capital no empreendimento. Em relação ao VPL estático, o aumento do valor da empresa foi de $52,49 \%$ com a utilização da opção de compra. No caso da opção de venda, o aumento foi insignificante, sendo mais vantajoso o investidor continuar com empreendimento, uma vez que, C > P. Comparando as duas opções, o investidor somente venderia o projeto se o comprador pagasse o valor do VPL TOR - compra, pois é o maior valor de retorno do projeto. Na hipótese do valor crítico do projeto ser de $\mathrm{R} \$ 11.789 .598,70$, os investidores poderiam realizar aportes no empreendimento, uma vez que, no curto prazo, não exerceriam a opção de venda, e sim a opção de compra, se o cenário econômico fosse favorável.

\subsection{APLICAÇÃO DO MODELO DE GESKE}

O modelo de Geske (1979) é uma solução para precificar opções compostas para mercados com taxa de juros constante e volatilidade do preço das ações semelhante ao preço do ativo. Neste caso, como não estamos tratando do mercado acionário, e sim, do mercado de bens e serviços, utiliza-se de valores da principal moeda estrangeira (dólar), uma vez que, as empresas sofrem influência da volatilidade. Outra característica do modelo é a utilização de um investimento inicial A, e por alguma hipótese há um adiamento do projeto, e neste caso, necessitaria de outro investimento inicial B, antes do investimento em produtividade, logo, aplicasse na existência de dois investimentos, antes de investir na produção para comercialização (Perlitz et al., 1999). 
A aplicação neste trabalho está ajustada para avaliação em opções reais por Kemna (1993), lembrando que esse modelo incorpora o investimento inicial, ao contrário do Black \& Scholes que precifica apenas a opção. O modelo de Geske não gera a opção de compra ou de venda como descrito acima, porém ele precifica o valor do retorno do projeto total a partir do segundo investimento. Contudo, nesta pesquisa, ele será utilizado caso haja adiamento na fase inicial. O modelo de Geske (1979) ajustado por Kemna (1993) será utilizado para efeito de comparação estocástica em Black \& Scholes (1973), uma vez que, ambos seguem as regras do movimento geométrico browniano para um crescimento de acordo com a distribuição normal.

Substituindo os dados da Tabela 1, nas Eqs. 9, 10 e 11;

$$
\begin{gathered}
\rho=(1 / 2) 0,5=0,7071 \\
h=\ln (22.185 .970,42 / 3.636 \cdot 189,83)+1 / 2 * 0,3^{2} * 2 / 0,3^{*} 2^{1 / 2}=4,4749 \\
k=\ln (22 \cdot 185.970,42 / 11 \cdot 789.598,70)+1 / 2 * 0,3^{2} * 1 / 0,3^{*} 1^{1 / 2}=2,2575
\end{gathered}
$$

Deve-se calcular os dados:

$$
\begin{array}{r}
N\left(k-\sigma t^{* 1 / 2}\right)=N\left(2,257474831-0,3 * 1^{1 / 2}\right)=N(1,95747483)=0,4749 \\
M(k, h ; \rho)=M(2,257474831,4,474863375 ; 0,707106781=0,9880 \\
M\left(k-\sigma t^{* 1 / 2}, k-\sigma t^{1 / 2} ; \rho\right)=M(1,9575,1,8332 ; 0,7071)=0,9731
\end{array}
$$

Ainda, determinar o valor crítico (Fc) da Eq. 12;

$$
\begin{gathered}
I=\left(R \$ 572.900,00+R \$ 572.900,00 *(1,14)^{1}+R \$ 3.636 .189,83\right)=R \$ 4.862 .195,83 \\
F c=R \$ 4.862 .195,83+R \$ 6.927 .402,87=R \$ 11.789 .598,70
\end{gathered}
$$

Utilizando-se o modelo de Geske (1979), ajustado por Kemna (1993) na Eq. 8 para uma opção composta, obtemos o valor presente;

$$
\mathrm{G}=\mathrm{R} \$ 16.019 .950,46
$$

Logo, o valor presente líquido em Geske:

$$
\mathrm{G}=\mathrm{R} \$ 16.019 .950,46-\mathrm{R} \$ 572.900,00_{\mathrm{t}=0}=\mathrm{R} \$ 15.447 .050,46
$$


A utilização dos dois modelos proposto pela pesquisa, exemplifica uma análise de investimento mais robusta, em relação a tradicional. O investidor munido de outras ferramentas, obtém um elenco de variáveis que podem auxiliar na tomada de decisão em relação ao empreendimento.

Tabela 2: Resultados Propostos

\begin{tabular}{l|c}
\hline \multicolumn{1}{c|}{ Método } & Valores $(\mathrm{R} \$)$ \\
\hline Valor Presente Líquido Tradicional & $\mathrm{R} \$ 13.198 .009,97$ \\
\hline Valor Presente Líquido TOR $_{\text {compra }}$ & $\mathrm{R} \$ 20.125 .412,84$ \\
\hline Valor Presente Líquido TOR & $\mathrm{R} \$ 13.198 .211,77$ \\
\hline Método de Geske & $\mathrm{R} \$ 15.447 .050,46$ \\
\hline
\end{tabular}

A flexibilidade administrativa dentro de uma abordagem probabilística permite ao investidor tomar decisões que, apenas com o valor presente líquido - VPL tradicional, não o permitia observar outras possibilidades com variáveis estocásticas, tempo de investimento e oscilações de mercado. O VPL produzido foi de R $\$ 13.198 .009,97$, logo, observando o valor presente líquido em TOR compras, percebe-se que é maior 52,49\% em relação ao tradicional. O valor presente líquido em TOR vendas, torna-se insignificante nesta pesquisa, porém, se a decisão fosse de venda, mesmo descoberto, o investidor poderia vender o empreendimento, uma vez que, em TOR vendas o valor é superior ao valor crítico de R\$ 11.789.598,70. Pelo método de Geske (1973) ajustado por Kemna (1993) o resultado foi R\$ 15.447.050,46, superior a 17\% em relação ao tradicional. Neste caso para o investidor, a melhor opção seria investir e continuar produzindo, onde no curto prazo, o VPL estará pagando um valor acima do valor crítico e, a luz da abordagem probabilística, nas três análises seguintes, o retorno do projeto estaria acima da análise tradicional. Algumas observações foram feitas na geração dos resultados:

- O modelo de Black \& Scholes somente poderá ser adotado para economias com volatilidade moderada. Testando os dados, pode-se observar que oscilação acima de $30 \%$, os dados tenderam a zero, perdendo sua significância e confiabilidade.

- A variável tempo possui uma alta sensibilidade de impacto no modelo, se postergar por três anos, o valor crítico do projeto ficaria maior que o VPL, a melhor estratégia seria adiar. Ao longo do tempo, o lucro apresentou rendimentos decrescentes. De acordo com Evatt et al. (2010) e Haque et al. (2014), a utilização da abordagem estocástica possui limitações de volatilidade, esse problema está associado ao sistema de Hamilton-Jacobi-Bellman de equações diferencial parcial, por isso, neste caso, é usual a utilização de métodos numéricos (Júnior e Mariano, 2020; Langrené et al., 2015).

- Em simulação de Monte Carlo utilizando a taxa livre de risco como variável estocástica apresentou comportamento semelhante ao movimento browniano. Esse método permite ao investidor antecipar decisões para mercado de alta volatilidade. 


\section{CONSIDERAÇÕES FINAIS}

A proposta inicial do trabalho foi analisar através do projeto financeiro a viabilidade de instalação de empresas de logística ou centro de distribuição na BR 493/RJ - arco metropolitano, onde foram utilizadas fluxo de caixa descontado (FCD) de uma empresa instalada no distrito industrial do município de Queimados, a 42 KM do porto de Itaguaí. Sabe-se que o investidor possui informações imperfeitas que auxiliam na tomada de decisão e, o aspecto financeiro é apenas um deles. Para verificar a existência de possibilidades ao investidor foram utilizadas abordagens probabilísticas e estocásticas que após as críticas ao FCD em relação ao caráter estacionário e irreversível desse método, opta-se no trabalho pela aplicação da Teoria das Opções Reais através dos métodos de Black \& Scholes (1973) e Geske (1979) ajustado por Kemna (1993).

A pesquisa demonstra possiblidades para a tomada de decisão com reversibilidade. indicando que a opção de compra (investimento em produtividade) possui um retorno financeiro maior que 50\% em relação ao VPL estático, pagando um valor acima do investimento inicial, classificado como coberto. A opção de venda está em descoberto, o investidor poderá retornar parte do investimento uma vez que, a opção de venda ou abandono está acima do valor crítico do projeto. Logo, utilizando as possibilidades de TOR em compra ou venda, o investidor obterá no mínimo o retorno do capital investido. O aspecto importante na análise de Geske é a aplicação direta do VPL, em uma abordagem do movimento browniano, uma vez que, o modelo incorpora o investimento inicial e o fluxo de caixa descontado em relação a interferências e mercados de alta volatilidade. O método de Geske apresenta um valor maior que o VPL estático e tradicional, aumentando as possibilidades nas decisões do investidor para cenários de adiamento do inicia de produção.

A maior contribuição do trabalho foram o desenvolvimento de alternativas para análise financeira de projetos em mercados de alta volatilidade. A aplicação apresentou boa performance para projeções no curto prazo, de maneira que ao longo do tempo, o método tornou-se menor eficiente. $\mathrm{O}$ teste de sensibilidade demonstrou resultados satisfatórios para $\sigma$ até 30\%, para economias com volatilidade acima, o método não é orientado. 


\section{REFERÊECIAS}

BLACK, F.; JENSEN, M. e SCHOLES, M. - The Capital Asset Pricing Model: Some Empirical Tests. Studies in the Theory of Capital Markets, 1972.

BLACK, F.; SCHOLES, M. The pricing of options and corporate liabilities. Journal of Political Economy, v.81, n.3, p.637-654, May/June 1973.

BLACK, F.; LITTERMAN, Robert. Global Portfolio Optimization. Financial Analysts Journal; Sep/Oct 1992; 48, 5; ABI/INFORM Global pg. 28

BLACK, F - Beta and Return. The Journal of Portfolio Management. 1993.

COPELAND, T; KOLLER, T e MURRIN, J - Valuation: Measuring and Managing the Value of Companies. McKinsey \& Company Inc, 1995.

COPELAND, T.; ANTIKAROV, A. Real Options: A Practitioner's Guide; Texere, New York, 2003. DIXIT, A.K.; PINDYCK, R.S. Investment under uncertainty. Princeton, New Jersey: Princeton University Press, 1994.

EVATT. G, JOHNSON. P, DUCK. P, and HOWELL. S. The measurement and inclusion of $a$ stochastic ore-grade uncertainty in mine valuations using pdes. IAENG International Journal of Applied Mathematics, 40 (4):1-7, 2010.

FAMA, E. F. e FRENCH, K. R. - The Cross-Section of Expected Stock Returns. The Journal of Finance. v.67, n. 2, Jun 1992.

GESKE, R. The valuation of compound option. Journal of Financial Economics, v.7, n.1, p.63-81, Mar. 1979.

HAQUE, M. TOPAL, E. and LILFORD. E. A numerical study for a mining project using real options valuation under commodity price uncertainty. Resources Policy, 39(1):115-123, 2014.

KEMNA, A.G.Z. Case studies on real options. Financial Management, v.22, n.3, p.259-270, Autumn 1993.

LEE, H. (2011). A Real Option Approach to Valuating Infrastructure Investments. MSc Thesis, KDI School of Public Policy and Management. Retrieved August 5, 2013.

LANGRENÉ. N, TARNOPOLSKAYA. T, CHEN. W, ZHU. Z, and COOKSEY, M. New regression Monte Carlo methods for high-dimensional real options problems in minerals industry. In Proceedings of the 21st International Congress on Modelling and Simulation (MODSIM 2015), 2015.

LINTNER, John (1965). The valuation of risk assets and the selection of risky investments in stock portfolios and capital budgets, Review of Economics and Statistics, 47 (1), 13-37.

JÚNIOR, W.A.M, MARIANO, F.P. Comparação dos métodos numéricos na soluçãoDe problemas da fluidodinâmica computacional. Latin American Journal of Development 2 (6), p.582-588, 2020. 
MINARDI, A.M.A.F. Teoria de opções reais aplicada a projetos de investimentos. Revista de Administração de Empresas (RAE-FGV), São Paulo, v.40, n.2, p.74-79, abr./jun. 2000.

MINARDI, A. M. A. F. Teoria das Opções aplicada a projetos de investimento. São Paulo: Atlas, 2004. PERLITZ, M., PESKE, T., SCHRANK, R. Real Option Valuation: The New Frontier in R\&D Project Evaluation, R\&D Management, v. 29, n. 3, p. 255-269, 1999.

RIBEIRO, J. X. P. Fusões e incorporações: estudo dos dados financeiros de uma cooperativa de crédito após processo de aglutinação. Latin American Journal of Development 2 (1), p. 24-39, 2020.

RIMA: Relatório de implantação do Arco Metropolitano do Rio de Janeiro BR-493/RJ-109. Consórcio Concremat. Julho de 2007.

ROLL, R. e ROSS, S. A. - On the Cross Sectional Relation between Expected Returns and Betas. The Journal of Finance. v. 49, n. 1, Mar 1994.

SANTOS, E. M., PAMPLONA, E. O. Teoria das Opções Reais: Aplicação em Pesquisa e Desenvolvimento (P\&D). 2o Encontro Brasileiro de Finanças, IBMEC, Rio de Janeiro, RJ, julho de 2002.

SANTOS, E. M., PAMPLONA, E. O. Teoria das Opções Reais: uma atraente opção no processo de análise de investimentos. Revista de Administração da USP - RAUSP, v.40, n.3, julho/setembro de 2005.

SHARPE, W. F. - Capital Asset Market Prices: A Theory of Market Equilibrium Under Conditions of Risk. The Journal of Finance, v. 19, n. 3, Set 1964.

SHARPE, W. F.; ALEXANDER, G. e BAILEY, J. - Investments. Prentice Hall, Inc. 1999.

TREYNOR, Jack L. Market Value, Time, and Risk. Unpublished manuscript. 1961.

TREYNOR, Jack L. Toward a Theory of Market Value of Risky Assets. Unpublished manuscript. A final version was published in 1999, in Asset Pricing and Portfolio Performance: Models, Strategy and Performance Metrics. Robert A. Korajczyk (editor) London: Risk Books, pp. 15-22. 1962.

TRIGEORGIS, L. The nature of options interactions and the valuation of investments with multiple real options. Journal of Financial and Quantitative Analysis, v.28, n.1, p.1-21, Mar. 1993. 